\title{
Optical ac coupling to overcome limitations in the detection of optical power fluctuations
}

\author{
Patrick Kwee, ${ }^{1, *}$ Benno Willke, ${ }^{1}$ and Karsten Danzmann ${ }^{1}$ \\ ${ }^{1}$ Max-Planck-Institut für Gravitationsphysik (Albert-Einstein-Institut) and Leibniz Universität Hannover, 30167 \\ Hannover, Germany \\ *Corresponding author: patrick.kwee@aei.mpg.de
}

Received April 7, 2008; revised May 26, 2008; accepted May 28, 2008; posted June 3, 2008 (Doc. ID 94787); published June 27, 2008

\begin{abstract}
A high-sensitivity detection method for optical power fluctuations is demonstrated based on photodetection in reflection of an optical resonator with a specific impedance matching. That resonator is used to reduce the carrier power reflected by the resonator while preserving the power fluctuation sidebands for frequencies above the resonator bandwidth. A sensitivity of $7 \times 10^{-10} \mathrm{~Hz}^{-1 / 2}$ for relative power fluctuations was achieved with only $3 \mathrm{~mA}$ of detected photocurrent and $99.6 \%$ of the power remained for downstream experiments. As in the widely used ac coupling of electrical signals, this technique overcomes dynamic-range limits and reduces detector noise associated with large carrier amplitudes of the optical field. (C) 2008 Optical Society of America

OCIS codes: $040.5160,230.5750$.
\end{abstract}

Applications, such as the detection of bright squeezed light, sensors in optical communications, or laser frequency stabilizations, set demanding requirements on the photodetectors used. In particular for laser power stabilizations as in interferometric gravitational wave detectors $[1,2]$ or experiments on the entanglement of macroscopic bodies [3] very sensitive detectors of power fluctuations are necessary.

Typically, photodiodes are used to measure the optical beam power by converting it into a photocurrent. The fundamental sensitivity limit in this traditional setup is given by the shot-noise of the photocurrent $I$. The linear spectral density of the relative power noise (RPN, often called RIN) by the shot-noise is proportional to $1 / \sqrt{I}$. To improve the sensitivity of the power detector, the detected photocurrent and therefore the laser power at the photodiode must be raised. This causes problems with photodiode saturation and the dynamic range of the readout electronics. A further problem that limits the so far most sensitive power detection in the frequency band from $10 \mathrm{~Hz}$ to $1 \mathrm{kHz}$ [4] at low frequencies $\approx 10 \mathrm{~Hz}$ is a (presumably photodiode internal) noise source associated with the large dc current that prevents a shotnoise limited sensitivity even at a $26 \mathrm{~mA}$ photocurrent $\left(3.5 \times 10^{-9} \mathrm{~Hz}^{-1 / 2}\right)$.

In this Letter we use a power detection method, which we call optical ac coupling. Conventionally, electrical ac coupling is used to measure small signals on top of large de signals or slowly varying ones. We utilize the reflection at an optical resonator to create a similar effect, where the signal is attenuated at low frequencies, for measuring laser power fluctuations. Power fluctuation sidebands are reflected at the resonator called ac coupling cavity (ACC) for frequencies above the resonator bandwidth, whereas the carrier is almost completely transmitted through the ACC. The same method was already used to, e.g., detect squeezed light [5], with far less reduction of the carrier power though.

0146-9592/08/131509-3/\$15.00
With a photocurrent of only $3 \mathrm{~mA}$ we achieved a sensitivity of $7 \times 10^{-10} \mathrm{~Hz}^{-1 / 2}$ for which one would usually need to detect a photocurrent of about $700 \mathrm{~mA}$. We applied this optical ac coupling to measure the power noise of a solid-state Nd:YAG nonplanar ring oscillator (NPRO) laser at $1064 \mathrm{~nm}$ in wavelength between $300 \mathrm{kHz}$ and $50 \mathrm{MHz}$. This is, to our knowledge, the most sensitive power noise measurement in the frequency band from 10 to $50 \mathrm{MHz}$.

In the future the optical ac coupling could be used to increase the power noise detection sensitivity for gravitational wave detectors even at low frequencies of a few hertz to build high-speed high-sensitivity photodetectors for optical communication links or to set up more noise-resistant power detections.

Consider the field amplitude $U_{\text {in }}$ of a laser beam with the average amplitude $U_{0}$ that is modulated at a frequency $f=\omega / 2 \pi$ with a modulation index $m \ll 1$ in the plane-wave model

$$
U_{\text {in }}=U_{0}\left(1+m e^{i \omega t}\right) \text {. }
$$

This input beam is coupled into a resonant optical resonator. Then the field amplitude $U_{\text {refl }}$ of the reflected beam is given by the sum of the directly reflected field and the field leaking out of the resonator

$$
U_{\text {refl }}=U_{0}\left(a+m e^{i \omega t}[1-(1-a) h(f)]\right),
$$

where $h(f)=1 /\left(1+i f / f_{0}\right)$ describes the approximated power fluctuation filter effect of the resonator with the bandwidth $f_{0}$. The amplitude reflectivity of the input coupling mirror is approximated by one assuming a high-finesse resonator. The parameter $a$ describes the impedance matching of the resonator. The resonator is undercoupled for $a \in(0,1]$, impedance matched for $a=0$, and overcoupled for $a \in[-1,0)$ [6].

The average power in reflection of the resonator is

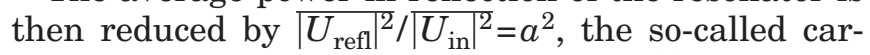
rier reduction. The transfer function $G(f)$ of relative 
power fluctuation from $\left|U_{\text {in }}\right|^{2}$ to $\left|U_{\text {reff }}\right|^{2}$ at the frequency $f$ is given by with $g=1 / a$

$$
G(f)=\frac{1-(1-a) h(f)}{a}, \quad|G(f)|=\sqrt{\frac{1+g^{2} \cdot f^{2} / f_{0}^{2}}{1+f^{2} / f_{0}^{2}}} .
$$

At high frequencies the transfer function phase depends on the sign of $a$ and thus on the impedance matching.

The maximum value $\left|G\left(f \gg f_{0}\right)\right| \approx|g|=1 /|a|$ depends on the carrier reduction and is reduced by nonresonant parasitic modes in the input beam, such as higher TEM modes or radio frequency modulation sidebands.

This transfer function $G(f)$ describes an optical amplification of power fluctuations for frequencies above the resonator bandwidth and can be used to build more sensitive power detectors.

In our experimental setup (Fig. 1) we used a singlefrequency continuous-wave NPRO as the laser. An electro-optic amplitude modulator (EOAM) along with a subsequent polarizing beam splitter was used to modulate the laser power. An optical ring resonator similar to the design described in [7] served as a mode cleaner (MC). This resonator had a finesse of $\approx 366$ and a bandwidth of $980 \mathrm{kHz}$. The Pound-Drever-Hall method [8] was used to stabilize the resonator length to the laser frequency using a piezoelectric element.

To measure power fluctuations with the photodetector HPD in a traditional setup $10 \%$ of the beam was sampled. About $90 \%$ of the power transmitted through the MC was directed to the resonator ACC. The geometry of the ACC was identical to the MC but mirrors with different reflectivity were used. Thus a finesse of $\approx 10,400$ and a bandwidth of $34 \mathrm{kHz}$ were measured. By tilting one mirror of the resonator the reflection angles of the resonator modes were changed to adjust the impedance matching. The

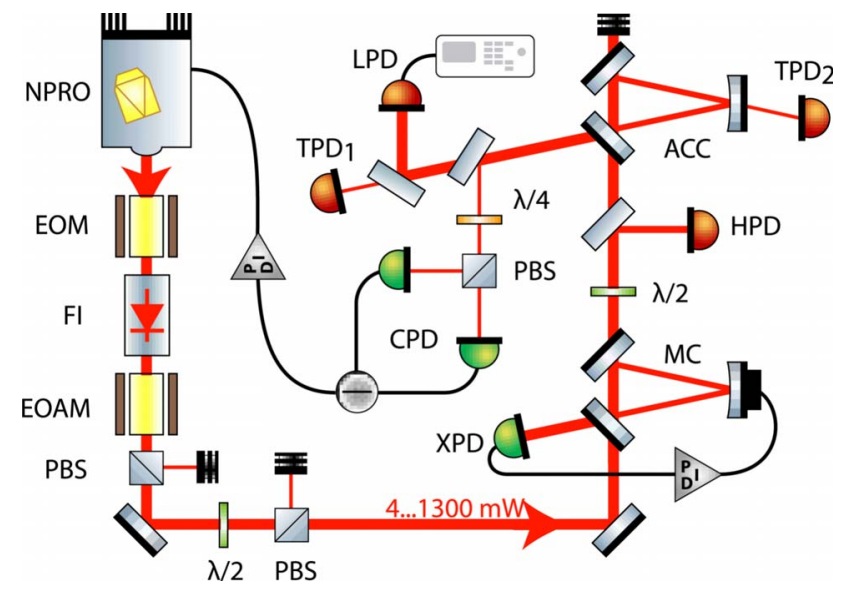

Fig. 1. (Color online) Experimental setup. The laser (NPRO) was locked to the resonator ACC. The power noise was measured with detector LPD in reflection of the ACC. Detector HPD was used to measure the power noise in a traditional way. EOM, electro-optic phase modulator; EOAM, electro-optic amplitude modulator; FI, Faraday isolator; PBS, polarizing beam splitter; $\lambda / 2, \lambda / 4$, wave plates.
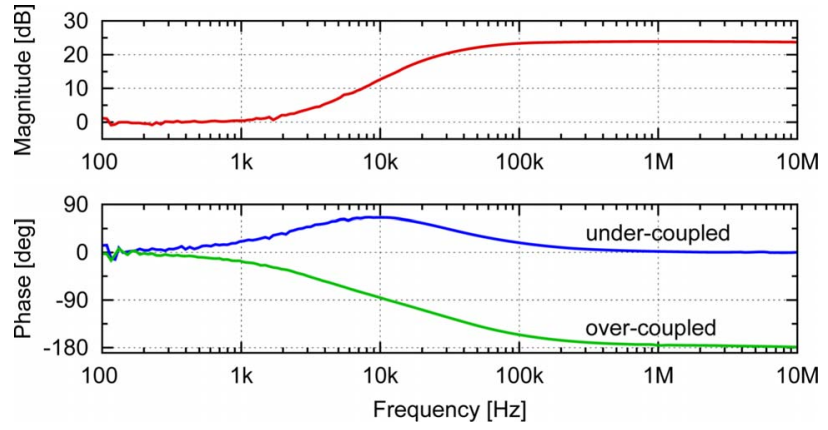

Fig. 2. (Color online) Measured transfer function $G(f)$ of RPN from the ACC input beam to the reflected beam using detectors HPD and LPD. The maximum gain was $|g|$ $=15.3$ with an upper corner frequency of $f_{0}=34 \mathrm{kHz}$. The phase depended on the impedance matching of the ACC.

Hänsch-Couillaud method [9] was used to stabilize the laser frequency to the ACC. About 99\% of the power reflected by the ACC was detected with the photodetector LPD at a photocurrent of $3 \mathrm{~mA}$.

The laser power in front of the ACC was about $900 \mathrm{~mW}$. For the main measurement the ACC was adjusted to be slightly undercoupled and along with parasitic modes about $0.4 \%$ of the impinging power was reflected.

The transfer function $G(f)$ from relative power fluctuations in front of the ACC to the fluctuations in the reflected beam was measured with a network analyzer and the detectors HPD and LPD. For this the laser power was modulated with the EOAM. The measurement was performed with a slightly undercoupled and then again with a slightly overcoupled ACC. The carrier reduction between the locked and unlocked resonator was measured with the detector $\mathrm{TPD}_{1}$.

The transfer function $G(f)$ (Fig. 2) was measured several times for an error estimation and agreed very well with the model using the parameters $f_{0}=34.4 \pm 0.3 \mathrm{kHz}$ and $|g|=15.3 \pm 0.3$. As predicted, the transfer function for the undercoupled and overcoupled cases differed only in their phases. A carrier reduction to $0.39 \%$ of the original power was measured that would yield $|g|=15.9$. The small discrepancy between this value and the measured one can be explained by a fraction of $0.035 \%$ power in parasitic modes. This power was estimated to be $\geqslant 0.02 \%$ using the modescan technique [10] and detector $\mathrm{TPD}_{2}$.

The ac coupled power noise of the laser was measured with detector LPD and a spectrum analyzer. The RPN of the laser was calculated with the dc signal of the detector and the transfer function $G(f)$. To verify the measurement the RPN was measured in a traditional setup with detector HPD. Finally the power in front of the MC was reduced to about $0.4 \%$, and the laser frequency was set off-resonant to the ACC. In this case the ACC acted like a highreflectivity mirror and the transfer function was measured as $G(f) \equiv 1$ as expected.

The measurement of the RPN [Fig. 3(c)] agreed within the measurement and calibration accuracy $(\approx 1 \mathrm{~dB})$ up to $\approx 4 \mathrm{MHz}$ with the traditional measurement [Fig. 3(b)]. At higher frequencies, the tradi- 


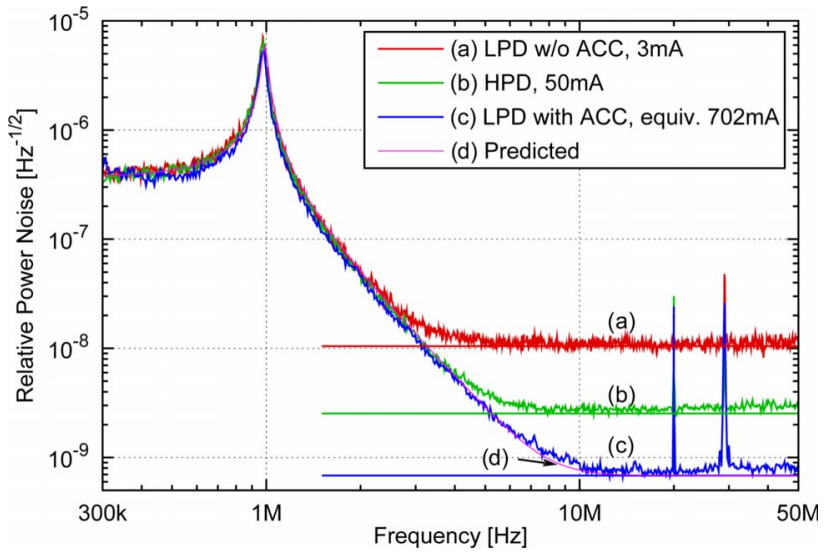

Fig. 3. (Color online) Measured power noise of the laser. The shot-noise limits for the different measurements are shown as horizontal lines (photocurrents are given in the legend). The sensitivity using the (c) optical AC coupling was increased by $G(f)$ compared to the same detector with (a) unlocked ACC. A traditional measurement with (b) detector HPD verified the measurement results. The signals at $20 \mathrm{MHz}$ were injected and those around $29 \mathrm{MHz}$ were due to the modulation for the Pound-Drever-Hall locking of the MC.

tional measurement was limited by shot-noise $\left(2.5 \times 10^{-9} \mathrm{~Hz}^{-1 / 2}, 50 \mathrm{~mA}\right.$ photocurrent $)$.

The frequency of the laser relaxation oscillation $(f=989 \mathrm{kHz})$ and the quality factor $(q=16)$ of the resonance were determined from measurement data below $2 \mathrm{MHz}$ to predict the $\mathrm{RPN}$ for frequencies $\geqslant 4 \mathrm{MHz}$ [Fig. 3(d)]. The measurement using the optical ac coupling agreed with the predicted RPN. From about $10 \mathrm{MHz}$ this measurement was limited by shot-noise at a level of $7 \times 10^{-10} \mathrm{~Hz}^{-1 / 2}$. Even though the detector LPD was operating at a photocurrent of only $3 \mathrm{~mA}$ a shot-noise limited sensitivity of $7 \times 10^{-10} \mathrm{~Hz}^{-1 / 2}$ was achieved for which in a traditional setup one would have to detect a photocurrent of $702 \mathrm{~mA}$. The comparison of the two measurements with detector LPD—once with locked [Fig. 3(c)] and once with unlocked ACC [Fig. 3(a)]—clearly showed the gain in sensitivity of this method by $G(f)$.

A power modulation signal was injected with the EOAM at $20 \mathrm{MHz}$ in all three measurements. This calibration signal had in all three measurements the same amplitude and thus verified the calibration.

In conclusion we can state that with the optical ac coupling the sensitivity of a photodetector for power fluctuations has been significantly increased for frequencies above the resonator bandwidth. The maximum gain $|g|$ is limited only by the controllability of the impedance matching and the power in parasitic modes. With the optical ac coupling it is possible to construct high-speed photodetectors using small pho- todiodes that can detect equivalent photocurrents that are more than 100 times larger than in traditional setups.

Furthermore only a fraction of the traditionally needed laser power is detected, whereby more power for subsequent experiments remains. This is especially an advantage for power stabilizations.

For gravitational wave detectors with kilometer baselines this method could be transferred for low frequency power stabilizations at a few hertz. The power recycling cavity [11] of such detectors that, e.g., in the case of Advanced LIGO (Laser Interferometer Gravitational-Wave Observatory) [12] has a bandwidth of $\approx 1 \mathrm{~Hz}$, could be used as ACC by proper adjustment of the impedance matching, e.g., with an etalon as the power recycling mirror.

The traditional detection of power noise below the $1 \times 10^{-8} \mathrm{~Hz}^{-1 / 2}$ level is prone to be limited by pointing fluctuations on the photodiode, stray light, or photodiode internal noise sources [4]. With the optical ac coupling a more noise-resistant power detection is possible since the photodetector would need a sensitivity of only $1 \times 10^{-8} \mathrm{~Hz}^{-1 / 2}$ to reach an equivalent sensitivity better than $1 \times 10^{-9} \mathrm{~Hz}^{-1 / 2}$.

This research was supported by the Deutsche Forschungsgemeinschaft and is part of Sonderforschungsbereich 407.

\section{References}

1. K. Somiya, Y. Chen, S. Kawamura, and N. Mio, Phys. Rev. D 73, 122005 (2006).

2. B. Willke, K. Danzmann, M. Frede, P. King, D. Kracht, P. Kwee, O. Punken, R. Savage, B. Schulz, F. Seifert, C. Veltkamp, S. Wagner, P. Weßels, and L. Winkelmann, Class. Quantum Grav. 25, 114040 (2008).

3. H. Müller-Ebhardt, H. Rehbein, R. Schnabel, K. Danzmann, and Y. Chen, Phys. Rev. Lett. 100, 013601 (2008).

4. F. Seifert, P. Kwee, M. Heurs, B. Willke, and K. Danzmann, Opt. Lett. 31, 2000 (2006).

5. G. Milburn, M. Levenson, R. Shelby, S. Perlmutter, R. DeVoe, and D. Walls, J. Opt. Soc. Am. B 4, 1476 (1987).

6. A. E. Siegman, Lasers (U. Science, 1986), Chap. 11.3.

7. B. Willke, N. Uehara, E. Gustafson, R. L. Byer, P. J. King, S. U. Seel, and R. L. Savage, Opt. Lett. 23, 1704 (1998).

8. R. W. P. Drever, J. L. Hall, F. V. Kowalski, J. Hough, G. M. Ford, A. J. Munley, and H. Ward, Appl. Phys. B 31, 97 (1983).

9. T. Hansch and B. Couillaud, Opt. Commun. 35, 441 (1980).

10. P. Kwee, F. Seifert, B. Willke, and K. Danzmann, Rev. Sci. Instrum. 78, 073103 (2007).

11. P. R. Saulson, Fundamentals of Interferometric Gravitational Wave Detectors (World Scientific, 1994), Chap. 12.7.

12. A. Weinstein, Class. Quantum Grav. 19, 1575 (2002). 